\title{
Comparative Assessment of Organic and Inorganic Tea Leaf Extract Feeding on Anxiety Behaviour Status of Colchicine Induced Rat Model of Alzheimer's Disease
}

\author{
Ananya Bagchi \\ Indian Institute of Technology Kharagpur \\ Dillip Kumar Swain ( $\square$ swain@agfe.iitkgp.ac.in ) \\ Indian Institute of Technology Kharagpur https://orcid.org/0000-0001-9883-3307 \\ Analava Mitra \\ Indian Institute of Technology Kharagpur
}

\section{Research Article}

Keywords: Tea polyphenols, Alzheimer's disease, anxiolytic behaviour, Corticosterone level

Posted Date: December 3rd, 2021

DOI: https://doi.org/10.21203/rs.3.rs-1126396/v1

License: (c) (1) This work is licensed under a Creative Commons Attribution 4.0 International License. Read Full License

Version of Record: A version of this preprint was published at Inflammopharmacology on March 9th, 2022. See the published version at https://doi.org/10.1007/s10787-022-00943-x. 


\section{Abstract}

Tea (Camellia sinensis) having anti-inflammatory, antioxidant, and free radical scavenging properties, may be beneficial to prevent the symptoms of neurodegenerative disorders like Alzheimer's disease (AD). In this present study, field experiments using the productive tea clone (TV25) with four nutrient management treatments were conducted during 2015 to 2017 in the research farm of Agricultural and Food Engineering Department, Indian Institute of Technology Kharagpur. The four nutrient management treatments were no application of fertilizer (control), organic fertilizer (OF), inorganic fertilizer (IF), and integration of OF and IF (IF+OF). The total phenolic content of tea leaves in terms of gallic acid equivalent (GAE) in control, OF, IF, IF+OF were $263 \pm 2.4 \mathrm{mg} / \mathrm{g}, 292 \pm 1.6 \mathrm{mg} / \mathrm{g}, 203 \pm 2.43 \mathrm{mg} / \mathrm{g}, 288.6 \pm 2.11$ $\mathrm{mg} / \mathrm{g}$, respectively. Tea leaf samples of these treatments were fed to the intracerebroventricular (ICV) colchicine administered rats. The animal study was double-blinded and randomized. Assessment of anxiety status was done for the rat model in an elevated open field with a novel object in two intervals (14-day and 21-day study). Anxiolytic behaviour with the lower corticosterone (CORT) level $(82 \mathrm{ng} / \mathrm{ml}$ ) was observed in ICV colchicine administered rat models of AD. After feeding of organically and inorganically grown tea extract $(20 \mathrm{mg} / \mathrm{kg})$ for 14 days and 21 days, it was found that the anxiolytic behaviour decreased with the increased concentration of serum CORT. However, organic tea showed greater increase in CORT level $(216.1 \mathrm{ng} / \mathrm{ml})$ as compared to inorganic tea $(214 \mathrm{ng} / \mathrm{ml})$. Thus, this study showed organic tea may act as a favourable agent or adjuvant in the improvement of the anxiolytic behaviour in rat model of $A D$.

\section{Introduction}

Alzheimer's disease (AD) is a neurodegenerative disorder. It is mainly distinguished by memory loss, language disability, poor learning, reasoning ability and dysfunction in cognition in elderly people. $A D$ is specified by gradual neuronal loss and generation of amyloid beta plaques $(A \beta)$, hyperphosphorylated tau proteins in brain (Glenner et al. 1983; Masters et al. 1985; Huang et al. 2009). Studies showed, generation of amyloid beta plaques, neurofibrillary tangles, tau protein, generation of reactive oxygen species and neuronal loss are the primary causes of AD (Bonda et al. 2009; Galasko et al. 2010; Schrag et al. 2013). Change in anxiety status is one of the most common syndromes of AD (Vloeberghs. 2007). Studies showed that plant food rich in phenolics and flavonoids can resist AD in many ways because of their antioxidant property (Muthaiyah et al. 2014). It has also been found that extract of tea, which is naturally rich in polyphenol and flavonoid content can reduce oxidative stress by inhibiting reactive oxygen species in cultured neurons associated with AD ( Park et al. 2008;Mishra et al. 2011). The polyphenol content and free radical scavenging properties of tea varies depending upon the field level nutrient management practices. It was found that organically grown tea is enriched with much better polyphenol content than tea grown inorganically (Bagchi et al. 2020).

For many years different types of animal models are considered to be important implements for developing many therapeutic models for human diseases (Benedikz et al. 2009). Gradual memory loss, dysfunction in cognition leading to poor learning and reasoning ability are the most commonly found 
characteristics of AD. Studies showed, varying anxiety status in rat model of AD depends on the preparatory process. For an example Raghavendra et al. (2008) showed anxiogenic behaviour in AD rats in an elevated plus maze (EPM) whereas Sil et al. (2015) has reported anxiolytic behaviour of AD rats in an open field space which is elevated with a novel object in the centre. It was found that intracerebroventricular (ICV) administration of colchicine to rats assists to a progressive neurodegeneration, cognitive impairment, neuronal loss and memory impairment. So, this type of model should be considered as a significant model of AD (Kumar et al. 2006; Kumar et al. 2007; Kumar et al. 2008; Kumar et al. 2010). Studies have shown that tea leaf extract contains several polyphenols and has antioxidant, anti-inflammatory properties (Khan and Mukhtar 2018). Besides, several studies related to the therapeutic effect of tea leaf extract on different diseases, very few studies have delineated on the Anti-Alzheimer's property of tea. Based on available literature, which showed that tea has antioxidant properties, it can be hypothesized that tea leaf extract should be effective to treat AD like pathology. In view of this, the present study mostly covered the anti-anxiolytic property of tea leaves grown under different field level nutrient management practices on rat models of AD. So far, the study demonstrating the anti-anxiolytic effect of organically and inorganically grown tea leaf extract on $A D$ animal model is very sparse. Therefore, the present study was conducted to evaluate the anti-anxiolytic effect of organically and inorganically grown tea to ICV colchicine injected AD rats.

\section{Materials And Methods 2.1 Tea leaf sampling}

The tea leaf samples as two leaves and a bud were collected in three commercial harvest time (May, July, and September) from different fertilizer management treatments in the years 2016 and 2017 from the tea garden of Agricultural and Food Engineering Department, Indian Institute of Technology, Kharagpur. Plucking of tea leaves depends on the flush of the leaves. The interval between successive plucking varied from 7-10 days. After plucking, the tea leaves were washed thoroughly and air dried in a drying chamber (Hot air oven) at $60^{\circ} \mathrm{C}$ for 6 hours (Chan et al. 2008; Palit et al. 2008). The dried samples were grounded in Wiley mill and passed through 80 mesh sieves. The dried samples were then stored in polythene packets for chemical analysis.

\subsection{Measurement of total phenolics in tea extracts}

The total phenolics content of tea samples at two leaves and a bud stage grown under different nutrient management practices were evaluated.

\subsubsection{Preparation of tea leaf extract}

The stored dried leaf samples were crushed thoroughly with mortar and pestle with deionized water. The extracts were centrifuged at $5000 \mathrm{rpm}$ for 20 minutes. The supernatant after centrifugation was collected after centrifugation and stored at $20^{\circ} \mathrm{C}$ for further experiments. 


\subsubsection{Estimation of total phenolics}

The total phenolics content in the tea leaf extract was estimated as per the procedure described in Singleton and Rasso (1965), using Folin-ciocalteu's phenol reagent (FCR). The oxidizing agent phosphomolybdate present in the Folin-ciocalteu's phenol reagent reacts with phenols present in the tea leaf extract and formed a blue colored complex. For calibration curve preparation, $1 \mathrm{ml}$ aliquots of 5, 10, $20,30,50$, and $75 \mu \mathrm{g} / \mathrm{ml}$ methanolic gallic acid solutions were mixed with $2.5 \mathrm{ml}$ of FCR which was diluted tenfold. To this reaction mixture, $2 \mathrm{ml}$ sodium carbonate $(75 \mathrm{~g} / \mathrm{l})$ was added. The absorption of this reaction mixture was measured after 5 minutes at $50^{\circ} \mathrm{C}$ at $765 \mathrm{~nm}$ with a UV/Visible spectrophotometer. One $\mathrm{ml}$ of tea leaf extract $(1 \mathrm{mg} / \mathrm{ml})$ was mixed with the reaction mixture and the absorption was taken for 5 minutes for the determination of the tea leaf polyphenols. The experiments were done in triplicate. The total content of the polyphenols in the tea leaf extract was calculated and expressed as gallic acid equivalent.

$$
c=c \cdot \frac{V}{m}
$$

where:

$\mathrm{C}=$ total concentration of the phenolic compounds, expressed as $\mathrm{mg} / \mathrm{g}$ in $\mathrm{GAE}$

$c=$ the concentration GAE expressed from the calibration curve

$V=$ Volume of the extract in $\mathrm{ml}$

$\mathrm{m}=$ the weight of plant methanolic extract expressed in $\mathrm{g}$

\subsection{Animal study}

\subsubsection{Experimental animals}

Healthy albino rats (Charles Foster strain) were selected for this experiment. Each of the experimental rat weighed 200-250 g (6-8 weeks of age). Total animals used were one hundred and thirty-five. Rats were kept individually in polypropylene animal cages. For the experimental animals the proper ethical guidelines were followed (Jaykaran et al.2011). The standard protocol used was accepted by the institutional animal ethics committee of Institute of Reproductive medicine IRM Kolkata, India (473/01/a/CPCSEA dated 5/9/2013). The rats were assigned in five groups with twenty-seven rats in each group in a random and blind folded manner. All animal model experiments were performed in Razabazar Science College, University of Calcutta (India) and in School of Medical Science and Technology, Indian Institute of Technology, Kharagpur (India)

\subsubsection{Preparation of Alzheimer's disease rat models}


For preparation of experimental rat models of $A D 2.5 \mu \mathrm{L}$ of artificial cerebrospinal fluid (ACSF) was taken. In $2.5 \mu \mathrm{L}$ ACSF $7.5 \mu \mathrm{g}$ colchicine was dissolved and was administered slowly for 5 minutes in the lateral ventricle of the experimental rats, to be treated as AD model. The lateral ventricle of both sides of the anaesthetized brain of the rats was approached stereotaxically (Kumar et al. 2006 ;Kumar et al. 2007; Kumar et al. 2010) through a steel cannula attached to a Hamilton syringe. Preparation of AD rat model by intracerebroventricular administration of colchicine is shown in Image 1.

The total rats were distributed into five groups with 27 rats in each group.

The groups are: Normal (Group 1): tea extract was fed without any ICV administration; Normal+ACSF (Group 2): ICV artificial cerebrospinal fluid (ACSF) was administered and tea extract was fed; AD rats +Tea (Group 3): tea extract was fed to ICV Colchicine administered rats; Untreated AD rats (negative control): tea extract was not fed to ICV colchicine administered rats; AD rats+ Celecoxib (positive control): the NSAID Celecoxib was given to ICV colchicine administered rats

Group 1, 2 and 3 were further subdivided into three subgroups with 9 rats in each subgroup. In each subgroup tea extract ( $20 \mathrm{mg} / \mathrm{kg}$ IP.) treatment of different nutrient management practices was given (Rezai Zadeh et al. 2005).

\subsubsection{Treatment schedule of drugs}

Standard fresh solutions of colchicine (St. Louis, MO, USA) were made at the start of each experiment. For ICV administration a $15 \mathrm{mg}$ dose of colchicine dissolved in ACSF should be given in a $5 \mathrm{~mL}$ injection volume. For therapeutic purpose, tea leaf extract $(2 \mathrm{~g}$ of fresh tea leaves macerated in $100 \mathrm{ml}$ of drinking water) was administered intraperitoneally (IP) at a dose of $20 \mathrm{mg} / \mathrm{kg}$ to Group 1, 2, and 3 for 21 days. The tea extract treatment was started from 4 days prior to the ICV administration of colchicine (Halawany et al. 2017). In group 5 rats (positive control) celecoxib $(20 \mathrm{mg} / \mathrm{kg}$ ) was given through IP. injection for 21 days starting from 4 days before the intracerebroventricular administration of colchicine (Halawany et al. 2017).

\subsubsection{Anti-anxiolytic property of tea extract}

The anxiety behaviour was assessed in an elevated open field space with an object in the centre (Ennaceur et al. 2006). The anxiety status was evaluated on 14th and 21 st day after ICV administration of colchicine to the AD rats. In each session of the test, all the experimental animals were used for 10 minutes of anxiety test. Rats were kept for 10 minutes to traverse the area of the open space. Different parameters of this behavioural analysis like the delay and frequency of entry to the inner area, amount of time spent in inner and outer area, delay and frequency of approach to the object area were assessed in each trial of the experiment. Experimental rats in elevated open field experiment are shown in Image 2.

\subsubsection{Serum Corticosterone}

About $1.5 \mathrm{ml}$ blood was taken from the heart of each anesthetized rat by a syringe for collection of the serum. With the blood serum the corticosterone (CORT) level was determined in each group of rats by 
using a commercially available kit.

\section{Results}

\subsection{Content of total phenolics}

Content of total phenolics in tea leaf extract was presented in gallic acid equivalent (GAE). The total phenolics content in tea leaves sampled in the months May, July and September in 2016 and 2017 are presented in Figure 1. In general, the total phenolics content of tea leaves were higher in July sampling than May and September in both years. The highest phenolic content of tea leaves was noted in OF treatment, which was comparable to IF+OF in most sampling and both were significantly higher than rest nutrient treatments throughout the sampling period. The IF treatment gave the lowest value of total phenolics content in tea leaves throughout the sampling. In July sampling, the values of total phenolics content in OF treatment were 290.4 $\pm 1.9 \mathrm{mg} / \mathrm{g}$ GAE in 2016 and $296.1 \pm 1.7 \mathrm{mg} / \mathrm{g}$ GAE in 2017.In general, the total content of phenolics was higher in second year than first year sampling.

Organic fertilizer; IF: Inorganic fertilizer; IF+OF:Integrated fertilizer; C: Control

Vertical lines in the line curves represent standard error values and the values with different letters are statistically significant at $\mathrm{P}<0.05$

\subsection{Anti-anxiolytic property of tea leaves grown organically and inorganically}

Anxiety behaviour in rat model was assessed as per the protocol described in Ennaceur et al. 2006.Different anxiety parameters as described in the subsection 2.3.4 were evaluated in each trial of the experiment.

\subsubsection{Delay/latency of entry of the rats to the inner area}

The data in Table 1 stated that the delay or latency of entry to the inner area was significantly higher in untreated AD rats (Group 4) as compared to the normal and sham rats (Groups 1 and 2) on both 14th and 21 st day. Chronic treatment with tea extract $(20 \mathrm{mg} / \mathrm{kg}$ IP.) of different nutrient management practices showed a significant decline of this parameter in $A D$ rats (Group 3 ) as compared to untreated $A D$ rats (Group 4). Among the subgroups of Group 3 rats, the subgroup, treated with organic tea extract (OF) showed maximum reduction in latency as compared to inorganic tea extract (IF) in both days. However, the celecoxib treated AD rats (Group 5) had significantly lower latency than the tea extracts treated group (Group 3).

Table 1 Effect of tea extract and celecoxib on Latency of entry to the inner area (min) of different experimental groups of rats on 14th and 21st day after ICV administration 


\begin{tabular}{|c|c|c|c|}
\hline Groups & Tea extract & $\mathrm{Da} 14^{\text {th }}$ & Day $21^{\text {th }}$ \\
\hline \multirow{3}{*}{ Normal rats } & IF & $0.11 \pm 0.013^{a}$ & $0.07 \pm 0.016^{a}$ \\
\hline & OF & $0.09 \pm 0.013^{a}$ & $0.05 \pm 0.01^{a}$ \\
\hline & $\mathrm{IF}+\mathrm{OF}$ & $0.096 \pm 0.007^{a}$ & $0.08 \pm 0.017^{a}$ \\
\hline \multirow{3}{*}{ Normal rats $+\mathrm{ACSF}$} & IF & $0.12 \pm 0.016^{a}$ & $0.1 \pm 0.03^{a}$ \\
\hline & OF & $0.07 \pm 0.01^{a}$ & $0.09 \pm 0.02^{a}$ \\
\hline & $\mathrm{IF}+\mathrm{OF}$ & $0.09 \pm 0.006^{a}$ & $0.07 \pm 0.01^{a}$ \\
\hline \multirow{3}{*}{$\mathrm{AD}$ rats $+\mathrm{Tea}$} & IF & $1.5 \pm 0.19^{d}$ & $1.19 \pm 0.2^{d}$ \\
\hline & OF & $1.25 \pm 0.1^{c}$ & $0.92 \pm 0.0 .76^{\circ}$ \\
\hline & $\mathrm{IF}+\mathrm{OF}$ & $1.53 \pm 0.133^{d}$ & $1.11 \pm 0.16^{d}$ \\
\hline \multicolumn{2}{|c|}{$\mathrm{AD}$ rats } & $5.9 \pm 0.3^{6}$ & $7.8 \pm 0.34^{e}$ \\
\hline \multicolumn{2}{|c|}{$\mathrm{AD}$ rats+Celecoxib } & $0.9 \pm 0.07^{b}$ & $0.44 \pm 0.04^{b}$ \\
\hline
\end{tabular}

OF: Organic fertilizer; IF: Inorganic fertilizer; IF+OF: Integrated fertilizer

Each value represents the mean \pm standard error (SE). Different superscripts present statistically significant values at $p<0.05$

The groups are: Normal (Group 1) where tea extract was fed without any ICV administration; Normal+ACSF (Group 2), where ICV artificial cerebrospinal fluid (ACSF) was administered and tea extract was fed; $A D$ rats +Tea (Group 3), where tea extract was fed to ICV Colchicine administered rats; Untreated $A D$ rats (negative control), where no tea extract was fed to ICV colchicine administered rats; $A D$ rats+ Celecoxib (positive control), Where the NSAID Celecoxib was given to ICV colchicine administered rats

\subsubsection{Frequency of entry to the inner area}

Frequency of entry to the inner area was lowered in untreated AD rats (Group 4) as compared to that of respective normal and sham rats (Groups 1 and 2) on 14th and 21st day after ICV administration (Figure 2). Treatment with tea extract ( $20 \mathrm{mg} / \mathrm{kg}$ IP.) of different nutrient management practices in Group 3 were comparable and resulted a significant increase to the frequency as compared to untreated AD rats (Group 4) on both 14th and 21st day. Moreover, this parameter was comparable in tea extract treated group of $A D$ rats (Group 3), Celecoxib treated $A D$ rats (Group 5), normal, and sham rats (Group 1 and Group 2) on 21 st day. Thus, this result is establishing the fact that treatment with tea extracts of different nutrient management practices is effective to ameliorate the anxiety status in AD rats.

OF:Organic fertilizer treatment, IF: Inorganic fertilizer, IF+OF:Integrated fertilizer

Each bar represents mean $\pm S E$ value, and the bars with different letters are statistically significant at $p<$ 0.05 
The groups are: Normal (Group 1): tea extract was fed without any ICV administration; Normal+ACSF (Group 2):ICV artificial cerebrospinal fluid (ACSF) was administered and tea extract was fed; AD rats +Tea (Group 3): tea extract was fed to ICV Colchicine administered rats; Untreated AD rats (negative control): tea extract was not fed to ICV colchicine administered rats; AD rats+ Celecoxib (positive control): the NSAID Celecoxib was given to ICV colchicine administered rats

\subsubsection{Amount of time spent in the inner area}

The Figure 3 stated that the total amount time spent in the inner area by the untreated AD rats (Group 4) were $3.16 \pm 0.21 \mathrm{~min}$ on 14 th day and $3.64 \pm 0.22 \mathrm{~min}$ on $21 \mathrm{st}$ day, which were significantly lower than rest groups of rats. Feeding tea extract ( $20 \mathrm{mg} / \mathrm{kg} \mathrm{IP.)} \mathrm{of} \mathrm{different} \mathrm{nutrient} \mathrm{management} \mathrm{practices} \mathrm{in} \mathrm{Group} 3$ showed a noteworthy increament in this parameter as compared to the untreated AD rats (Group 4). The tea extract treatments of different nutrients management did not differ significantly in increasing the amount of time spent in group 3 rats. This parameter in AD rats + Tea (group 3) was comparable with celecoxib treated $A D$ rats (Group 5 ) on the day 14th, though the latter group of rats spent significantly higher time than the former on 21 st day.

OF:Organic fertilizer treatment, IF: Inorganic fertilizer, IF+OF:Integrated fertilizer

Each bar represents mean $\pm S E$ value, and the bars with different letters are statistically significant at $\mathrm{P}<0.05$

The groups are: Normal (Group 1): tea extract was fed without any ICV administration; Normal+ACSF (Group 2):ICV artificial cerebrospinal fluid (ACSF) was administered and tea extract was fed; AD rats +Tea (Group 3): tea extract was fed to ICV Colchicine administered rats; Untreated AD rats (negative control): tea extract was not fed to ICV colchicine administered rats; $A D$ rats+ Celecoxib (positive control): the NSAID Celecoxib was given to ICV colchicine administered rats

\subsubsection{Amount of time spent in the outer area}

Figure 4 stated that the amount of time spent in the outer area was remarkably higher in untreated $A D$ rats (Group 4) on both 14th and 21st day as compared to the other experimental group of rats. Feeding of tea extract $(20 \mathrm{mg} / \mathrm{kg} \mathrm{IP})$ of different nutrient management in group 3 rats significantly decreased this parameter as compared to the untreated AD rats (group 4). On 14th day, this parameter was comparable between tea extract fed AD rats (Group 3) and celecoxib treated AD rats (Group 5). However, on 21st day, the group 5 rats spent significantly lower time in outer area than the group 3 rats. The tea extract of different nutrients management showed similar effect in decreasing the time spent by rats in outer area.

OF:Organic fertilizer treatment, IF: Inorganic fertilizer; IF+OF:Integrated fertilizer

Each bar represents mean $\pm S E$ value, and different letters showed statistical significance at $p<0.05$

The groups are: Normal (Group 1): tea extract was fed without any ICV administration; Normal+ACSF (Group 2): ICV artificial cerebrospinal fluid (ACSF) was administered and tea extract was fed; AD rats 
+Tea (Group 3): tea extract was fed to ICV Colchicine administered rats; Untreated AD rats (negative control): tea extract was not fed to ICV colchicine administered rats; AD rats+ Celecoxib (positive control): the NSAID Celecoxib was given to ICV colchicine administered rats

\subsubsection{Delay/latency of first approach to the object area}

The delay/ latency of first approach to the object area was highest ( $8.48 \pm 0.19 \mathrm{~min}$ on day 14 th and 9.68 \pm 0.17 min on day $21 \mathrm{st}$ ) in untreated $A D$ rats (Group 4), which was significantly higher than rest groups on 14th as well as on 21st day after ICV administration of Colchicine (Figure 5). The latencies were $(4.75 \pm 0.19 \mathrm{~min}, 4.6 \pm 0.13 \mathrm{~min}, 4.67 \pm 0.12 \mathrm{~min})$ and $2.9 \pm 0.17 \mathrm{~min}$, respectively on 14 th day and $(4.05 \pm 0.11$ min, $3.9 \pm 0.1 \mathrm{~min}, 4.05 \pm 0.13 \mathrm{~min}$ ) and $2.32 \pm 0.2 \mathrm{~min}$ respectively on $21 \mathrm{st}$ day in tea extract treated $A D$ rats (Group 3 ) and celecoxib treated $A D$ rats (Group 5), which were significantly higher than normal and normal+ACSF rats (Group 1 and 2). Feeding of tea extracts (20 mg/kg IP.) of different nutrient management did not bring any significant variation in the latency value among the Group 3 rats. However, the celecoxib treated AD rats (Group 5) showed a significant reduction of this parameter over tea extract treated $A D$ rats (Group 3 ) on both days (day 14 th and day 21 st ).

OF:Organic fertilizer treatment, IF: Inorganic fertilizer, IF+OF:Integrated fertilizer

Each bar represents mean $\pm S E$ value, and different letters in the bars showed statistical significance at $p<$ 0.05

The groups are: Normal (Group 1): tea extract was fed without any ICV administration; Normal+ACSF (Group 2): ICV artificial cerebrospinal fluid (ACSF) was administered and tea extract was fed; AD rats +Tea (Group 3): tea extract was fed to ICV Colchicine administered rats; Untreated AD rats (negative control): tea extract was not fed to ICV colchicine administered rats; AD rats+ Celecoxib (positive control): the NSAID Celecoxib was given to ICV colchicine administered rats

\subsubsection{Frequency of approach to the object area}

Figure 6 revealed that the frequency of approach to the object area was lowest ( $3.42 \pm 0.19$ on day 14 th and $2.82 \pm 0.15$ on day $21 \mathrm{st}$ ) in untreated $A D$ rats (Group 4) and this was remarkably lower than rest of the rat groups on both days. Feeding tea extracts $(20 \mathrm{mg} / \mathrm{kg}$ IP) of different nutrient management practices showed a significant increase in the frequencies in group 3 rats as compared to the untreated $A D$ rats (Group 4) on both 14th and 21st day, though the values were significantly lower than normal rats (Group 1 and 2). The tea extracts of different nutrients management were comparable in increasing the frequency in group 3 rats. The Celecoxib treated rats (Group 5) showed a significant increase in this parameter as compared to the tea extract treated AD rats (Group 3) on both 14th and 21 st day and this was also comparable to normal rats on 14th day.

OF:Organic fertilizer treatment, IF: Inorganic fertilizer; IF+OF:Integrated fertilizer

The bars in the figure represent mean $\pm S E$ values, and different letters in the bars showed statistical significance at $p<0.05$ 
The groups are: Normal (Group 1): tea extract was fed without any ICV administration; Normal+ACSF (Group 2): ICV artificial cerebrospinal fluid (ACSF) was administered and tea extract was fed; AD rats +Tea (Group 3): tea extract was fed to ICV Colchicine administered rats; Untreated AD rats (negative control): tea extract was not fed to ICV colchicine administered rats; AD rats+ Celecoxib (positive control): the NSAID Celecoxib was given to ICV colchicine administered rats

\subsubsection{Serum Corticosterone Level}

The serum corticosterone (CORT) level in untreated AD rats (Group 4) were $82 \pm 2.06 \mathrm{ng} / \mathrm{ml}$ on 14th day and $79.6 \pm 3.19 \mathrm{ng} / \mathrm{ml}$ on $21 \mathrm{st}$ day, which were significantly lower than rest group of rats (Figure 7). The tea extracts $(20 \mathrm{mg} / \mathrm{kg}$ IP) treatment of different nutrient management practices in group 3 rats showed a significant improvement in total serum CORT level as compared to the untreated AD group (Group 4). Among the different nutrient managements, OF and IF+OF were better effective on 14th day and all (OF, $\mathrm{IF}+\mathrm{OF}$ and IF) were comparable on 21st day in increasing this parameter in Group 3 rats. The Celecoxib treated $A D$ rats (Group 5) had significantly higher serum CORT level as compared to the tea extract treated $A D$ rats (Group 3) on 21st day, but they were comparable on 14th day.

OF: Organic fertilizer treatment, IF: Inorganic fertilizer, IF+OF: Integrated fertilizer.

The bars in the figure represent mean $\pm S E$ values, and different letters in the bars showed statistical significance at $p<0.05$

The groups are: Normal (Group 1): tea extract was fed without any ICV administration; Normal+ACSF (Group 2): ICV artificial cerebrospinal fluid (ACSF) was administered and tea extract was fed; AD rats +Tea (Group 3): tea extract was fed to ICV Colchicine administered rats; Untreated AD rats (negative control): tea extract was not fed to ICV colchicine administered rats; AD rats+ Celecoxib (positive control): the NSAID Celecoxib was given to ICV colchicine administered rats

\section{Discussion}

ICV colchicine administered rat models of AD produced amyloid $\beta$ plaques in different areas of brain leading to neurodegeneration showing resemblance with AD pathology (Sil et al. 2015). Several studies showed that memory dysfunction in ICV colchicine administered rat models of AD exhibited comparability with AD patients (Kumar et al. 2002; Kumar et al. 2006; Kumar et al. 2007). Thus, ICV colchicine administered animal model is contemplated as a potent model of $A D$ by many researchers and has been taken as a model for the discovery of new therapeutic agents for $A D$ (Kumar et al. 2002; Kumar et al. 2006; Kumar et al.2007; Pitchaimani et al.2012). Therefore, rat models of AD prepared from ICV colchicine administration is relevant for studying different behavioural and pathological characteristics of AD. Neuroinflammation and oxidative stress due to free radical damage are most common causes of AD. Tea catechins can enhance the resistance of the cells to oxidative stress by its free radical scavenging and iron chelating property (Salah et al.1995;Lin et al.1998). Thus special interest should be given to the therapeutic effects of nutritional antioxidants and free radical scavengers in preventing 
neurodegenerative diseases (Gotz et al.1990; Halliwell.1992; Mandel et al. 2004; Weinreb et al.2004). AD patients have been reported with anxiety along with dementia for a long period of time. Though most of the animal models of AD showed memory impairment, inconsistency with anxiety behaviour can be seen to some extent. This inconsistency in anxiety behaviour is affected not only by different animal models of $A D$ but also by the method of study (Ennaceur et al.2006). The results of the present study exhibited different parameters of anxiety status in rat models of $A D$. This result demonstrates anxiolytic behaviour in group 4 rats (untreated $A D$ rats). But the anxiolytic behaviour was reversed after feeding with tea extract for 14 and 21 days. Thus, the result of the present study shows improvement in the anxiolytic behaviour in $A D$ rat models after treatment with organic and inorganic tea extract treatment. It was also found that organic tea extract treatment showed better improvement in anxiolytic behaviour in rat models of $A D$ than inorganic tea extract treatment. The anxiolytic behaviour in the untreated $A D$ rats (group 4) rats may be the cumulative effect of the damaged function of neuronal areas in brain which were contrived by colchicine administration. The recovery from neurodegeneration of these brain areas after chronic feeding of tea extract may be the reason behind the improvement of anxiolytic behaviour presented in the present study. The major catechins of tea leaves can traverse the blood brain barrier (Weinreb et al. 2009) and can protect colchicine induced neurodegeneration. The decreased serum corticosterone level was seen in the untreated AD rats and the feeding of $20 \mathrm{mg} / \mathrm{kg}$ body weight of organic and inorganic tea leaf extract stemmed in almost complete recovery of corticosterone on 14th and 21 st days of study. This convalescence of corticosterone in tea extract treated AD rats can be supported by the recovery from anxiolytic behaviour. The hypothalamo-hypophysial-adrenal (HPA) axis which is mainly required in the regulation of CORT secretion, may be non-functional in untreated AD rats due to the colchicine produced damage of the neuronal cells and free radical generated oxidative stress (Herman et al. 2016). Hindrance from this neuronal and free radical damage by tea polyphenols may be helpful to recuperate the normal activity of the HPA axis which is corroborated by this study. Earlier studies demonstrated that organic tea has higher amount of polyphenol content and better antioxidant property as compared to tea leaves grown under inorganic nutrient management practice (Bagchi et al.2020). In this present study it was found that organic tea can be more effective in ameliorating the anxiolytic behaviour in rat model of $A D$ as compared to tea leaves grown under inorganic fertilizer management. Thus it can be stated that this study is the first of its category which revealed the comparison of the anxiety status in rat models of $A D$, treated with tea leaf extracts grown under organic and inorganic nutrient management practices.

\section{Conclusions}

Tea (Camellia sinensis) grown under organic nutrient management resulted in improved quality as total phenolics content increased by approximately $30 \%$ as compared to inorganic nutrient management in subtropical climate. The July harvesting under high humidity and sufficient water availability could give better tea quality as compared to May and September harvest. Accumulation of the secondary plant metabolites i.e., EGC, EC, EGCG, and ECG in tea leaves were increased with organic nutrients, but decreased with inorganic nutrients management as compared to no fertilizer application. In animal model 
experiment, feeding rats with organic tea leaf extract acted as a favourable adjuvant or pharmacological agent to alleviate the anxiolytic symptoms of $A D$ in the rats. Moreover, the effectiveness of the organic tea extract was comparable with the traditional nonsteroidal anti-inflammatory drug 'celecoxib' for improvement in the anxiolytic behaviour of the AD rats.

\section{Declarations}

\section{Acknowledgements}

The authors would like to duly acknowledge the Indian Institute of Technology Kharagpur for the financial support and the laboratory facilities in School of Medical Science Technology and Agricultural and Food Engineering Department for conducting the experiments. Also the experimental facilities in Razabazar Science College (University of Calcutta, India) are acknowledged.

\section{Ethical standards}

For animal experiments, guidelines of CPCSEA, were followed. The ethical standard for animal experiments was approved by the animal ethical committee of the Institute of Reproductive Medicine (IRM) Kolkata, India (473/01/a/CPCSEA dated 5/9/2013).

\section{Conflict of interest}

The authors declare no conflict of interest among themselves.

\section{Data Availability}

The datasets generated during the current study are available.

\section{References}

1. AlAttar AM, Zarib TA (2010) Influences of crude extract of tea leaves, Camellia sinensis, on streptozotocin diabetic male albino mice. Saudi J. Biol. Sci.7:295-301. https://doi.org/10.1016/j.sjbs.2010.05.007

2. Bagchi A, Swain DK, Mitra A (2020) Neuroprotective effect of organic and inorganically grown tea on oxidative damage in rat model of Alzheimer's disease. Adv Tradit Med 20:439-450. https://doi.org/10.1007/s13596-020-00428-8

3. Benedikz E, Kloskowska E, Winblad B (2009) The rat as an animal model of Alzheimer's disease. J Cell Mol Med 13:1034-42. 1111/j.1582-4934.2009.00781.x

4. Bonda DJ, Wang X, Perry G, Nunomura A, Tabaton M, Zhu X, Smith MA (2010) Oxidative stress in Alzheimer disease:A possibility for prevention. Neuropharmacology 59:290-294. 10.1016/j.neuropharm.2010.04.005 
5. Chan E W C, Lim YY, Wong LF et al. (2008) Antioxidant and tyrosinase inhibition properties of leaves and rhizomes of ginger species. Food Chem.109, 477-483.

https://doi.org/10.1016/j.foodchem.2008.02.016.

6. Ennaceur A, Michalikova S, Chazot PL (2006) Models of anxiety: responses of rats to novelty in an open space and an enclosed space Behav Brain Res 171:26-49. 1016/j.bbr.2006.03.016

7. Galasko D, Montine TJ (2010) Biomarkers of oxidative damage and inflammation in Alzheimer's disease. Biomark Med 4:27-36. 2217/bmm.09.89

8. Glenner GG (1983) Alzheimer's disease. The commonest form of amyloidosis. Arch Pathol Lab Med. 107:281-282

9. Gotz ME, Freyberger A, Riederer P (1990) Oxidative stress: a role in the pathogenesis of Parkinson's disease. J Neural Transm Suppl.29:241-249. 1007/978-3-7091-9050-0_23

10. Halliwell B (1992) Reactive oxygen species and the central nervous system. J Neurochem.59:16091623. 1111/j.1471-4159.1992.tb10990.x

11. Halawany AM, Sayed NS, Abdallah HM, Dine RS (2017) Protective effects of gingerol on streptozotocin induced sporadic Alzheimer's disease: emphasis on inhibition of beta amyloid, cox2, alpha, beta secretases and aph1a. Sci Rep.7: 2902. 1038/s41598-017-02961-0

12. Herman JP, McKlveen JM, Ghosal S, Kopp B, Wulsin A, Makinson R, Scheimann J, Myers B (2016) Regulation of the Hypothalamic-Pituitary-Adrenocortical Stress Response. Compr Physiol. 6:603-621. doi: 10.1002/cphy.c150015.

13. Huang HC, Jiang ZF (2009) Accumulated amyloid-beta peptide and hyperphosphorylated tau protein: Relationship and links in Alzheimer's disease. J Alzheimers Dis 16:15-27. 3233/JAD-2009-0960

14. Jaykaran, Yadav P, Kantharia ND (2011) Reporting of method of animal sacrifice in articles published in Indian journals. J Pharmacol Pharmacother. 2: 125- 127. 4103/0976-500X.81912

15. Khan N, Mukhtar H (2018) Tea Polyphenols in Promotion of Human Health. Nutrients 11:39. 3390/nu11010039

16. Kumar A, Dogra S, Prakash A (2010) Protective effect of naringin, a citrus flavonoid, against colchicine induced cognitive dysfunction and oxidative damage in rats. J Med Food 13: 976-984. 1089/jmf.2009.1251

17. Kumar A ,Segal N ,Padi SS,Naidu PS(2008) Protective effect of quercetin against ICV colchicine induced cognitive dysfunctions and oxidative damage in rats Phytother Res 22:1563-1569. 1002/ptr.2454

18. Kumar A ,Segal N ,Padi S S , Naidu PS (2007) Effect of curcumin on intracerebroventricular colchicine-induced cognitive impairment and oxidative stress in rats J Med Food 10:486-494. 1089/jmf.2006.076

19. Kumar A,Segal N,Padi SS,Naidu PS (2006) Differential effects of cyclooxygenase inhibitors on intracerebroventricular colchicine-induced dysfunction and oxidative stress in rats Eur J Pharmacol 551:58-66. 10.1016/j.ejphar.2006.08.076 
20. Lin AM, Chyi BY, Wu LY, Hwang LS, Ho LT (1998)The antioxidative property of green tea against ironinduced oxidative stress in rat brain. Chin J Physiol.41:189-194.

21. Mandel S,Weinreb O,Amit T, Youdim MB (2004) Cell signalling pathways in the neuroprotective actions of the green tea polyphenol (-)-epigallocatechin-3-gallate: implications for neurodegenerative diseases. J Neurochem 88:1555-1569. 10.1046/j.1471-4159.2003.02291

22. Masters CL, Simms G, Weinman NA, Multhaup G, McDonald BL, Beyreuther K (1985) Amyloid plaque core protein in Alzheimer disease and Down syndrome. Proc Natl Acad Sci U S A. 82:4245-4249. 1073/pnas.82.12.4245

23. Matar MA, Zohar J, Kaplan Z, Cohen H (2009) Alprazolam treatment immediately after stress exposure interferes with the normal HPA-stress response and increases vulnerability to subsequent stress in an animal model of PTSD Eur Neuropsychopharmacol 19: 283-295.

10.1016/j.euroneuro.2008

24. Mishra, S, Mishra M, Seth P, Sharma SK (2011) Tetrahydro curcumin confers protection against amyloid beta-induced toxicity. Neuroreport 22: 23-27. 1097/WNR.0b013e328341e141

25. Muthaiyah B, Essa M M, Lee M, Chauhan V, Kaur K, Chauhan A (2014) Dietary Supplementation of Walnuts Improves Memory Deficits and Learning Skills in Transgenic Mouse Model of Alzheimer's Disease. Alzheimer's Dis 42: 1397-1405. 10.3233/JAD-140675

26. Palit S, Ghosh BC, Dutta Gupta S, Swain DK (2008)Studies on tea quality grown through conventional and organic management practices: its impact on antioxidant and antidiarrhoeal activity. Trans ASABE 51: 2227- 2238. 13031/2013.25376

27. Pitchaimani V, Arumugam S, Thandavarayan RA (2012) Nootropic activity of acetaminophen against colchicine induced cognitive impairment in rats. J Clin Biochem Nutr 50(3):241-244. 3164/jcbn.11-73

28. Raghavendra M, Maiti R, Kumar S, Acharya SB (2008) Role of Ocimum sanctum in the experimental model of Alzheimer's disease in rats.Int. J. Green Pharm 3: 6-15. 10.4103/0973-8258.49368

29. Rezai-Zadeh K, Shytle D, Sun, N et al.(2005).Green tea epigallocatechin3gallate (EGCG) modulates amyloid precursor protein cleavage and reduces cerebral amyloidosis in Alzheimer transgenic mice. J Neurosci 25:8807-8814. 1523/JNEUROSCI.1521-05.2005.

30. Salah N, Miller NJ, Paganga G, Tijburg L,Bolwell G P, Rice- Evans C(1995) Polyphenolic flavanols as scavengers of aqueous phase radicals and as chain-breaking antioxidants. Arch Biochem Biophys.322:339-346. 1006/abbi.1995.1473

31. Schrag M, Mueller C, Zabel M., Crofton A, Kirsch WM, Ghribi O, Squitti R, Perry G (2013) Oxidative stress in blood in Alzheimer's disease and mild cognitive impairment: A meta-analysis. Neurobiol Dis. 59:100-110. 1016/j.nbd.2013.07.005

32. Sil S,Ghosh TK (2015) Amelioration of anxiolytic behavior in intracerebroventricular colchicine injected rats by naproxen. Asian J. Pharm. Clin. Res 8(5):189-196. https://innovareacademics.in/journals/index.php/ajpcr/article/view/7230

33. Singleton VL, Rossi JA (1965) Colorimetry of total phenolics with phosphomolybdic-phosphotungstic acid reagents. Am J Enol Vitic 16: 144158. 
34. Vloeberghs E, Van Dam D, Franck F, Staufenbiel M, De Deyn PP (2007) Mood and male sexual behaviour in the APP23 model of Alzheimer's disease. Behav Brain Res 180:146-151. 1016/j.bbr.2007.03.002

35. Weinreb O,Mandel S, Amit T, Youdim MB (2004) Neurological mechanisms of green tea polyphenols in Alzheimer's and Parkinson's diseases. J Nutr Biochem. 15:506-516. 1016/j.jnutbio.2004.05.002

Figures

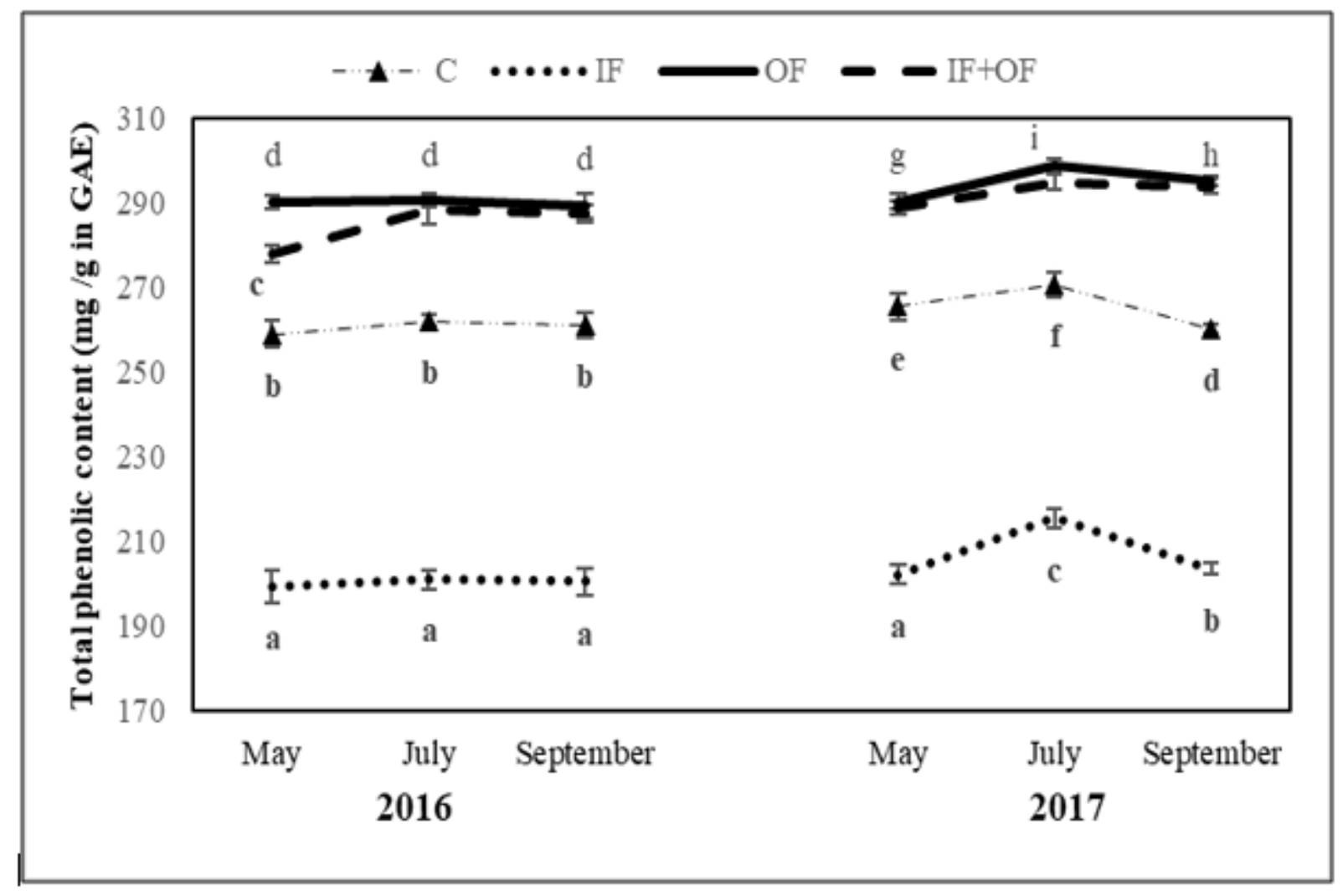

Figure 1

Effect of organic and inorganic fertilizer management and sampling months on total phenolic content of tea leaves in the years 2016 and 2017 


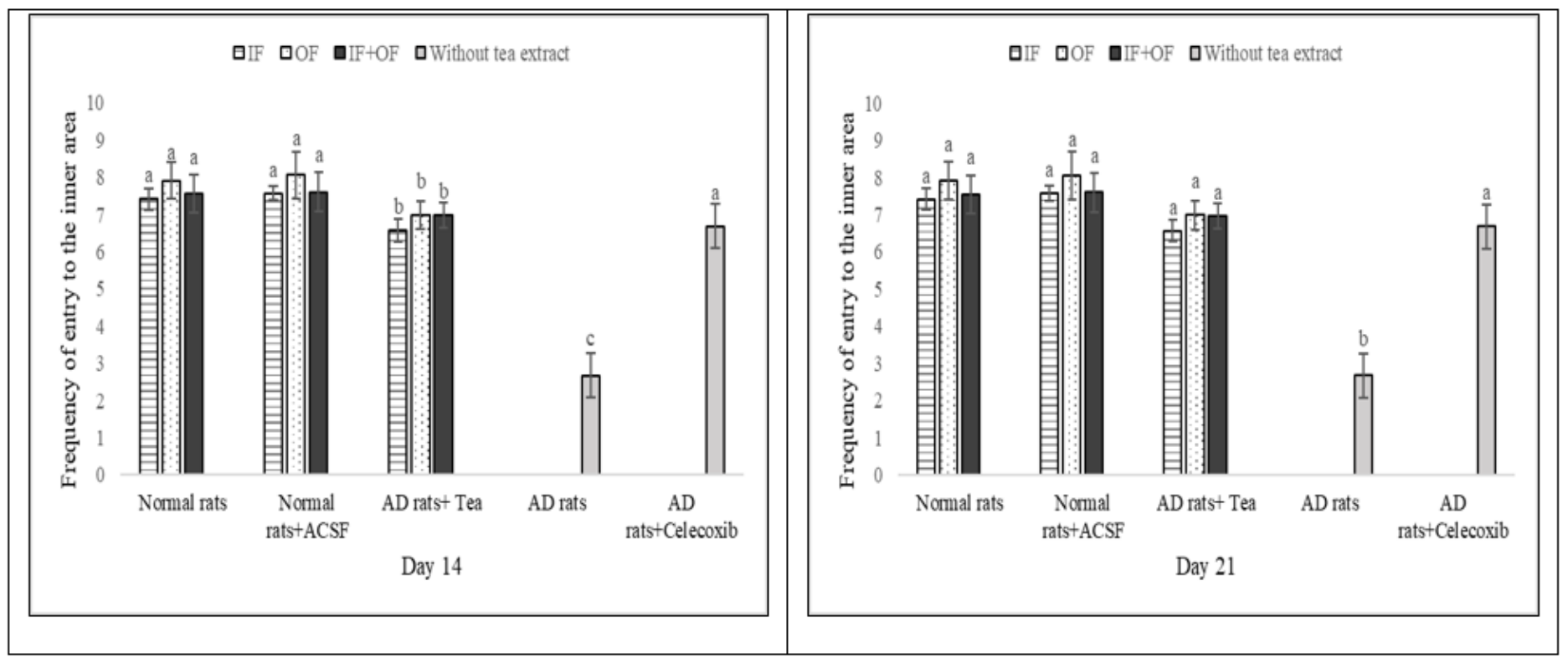

Figure 2

Impact of tea extracts of different field level nutrient management and celecoxib on frequency of entry to the inner area in different experimental groups of rats

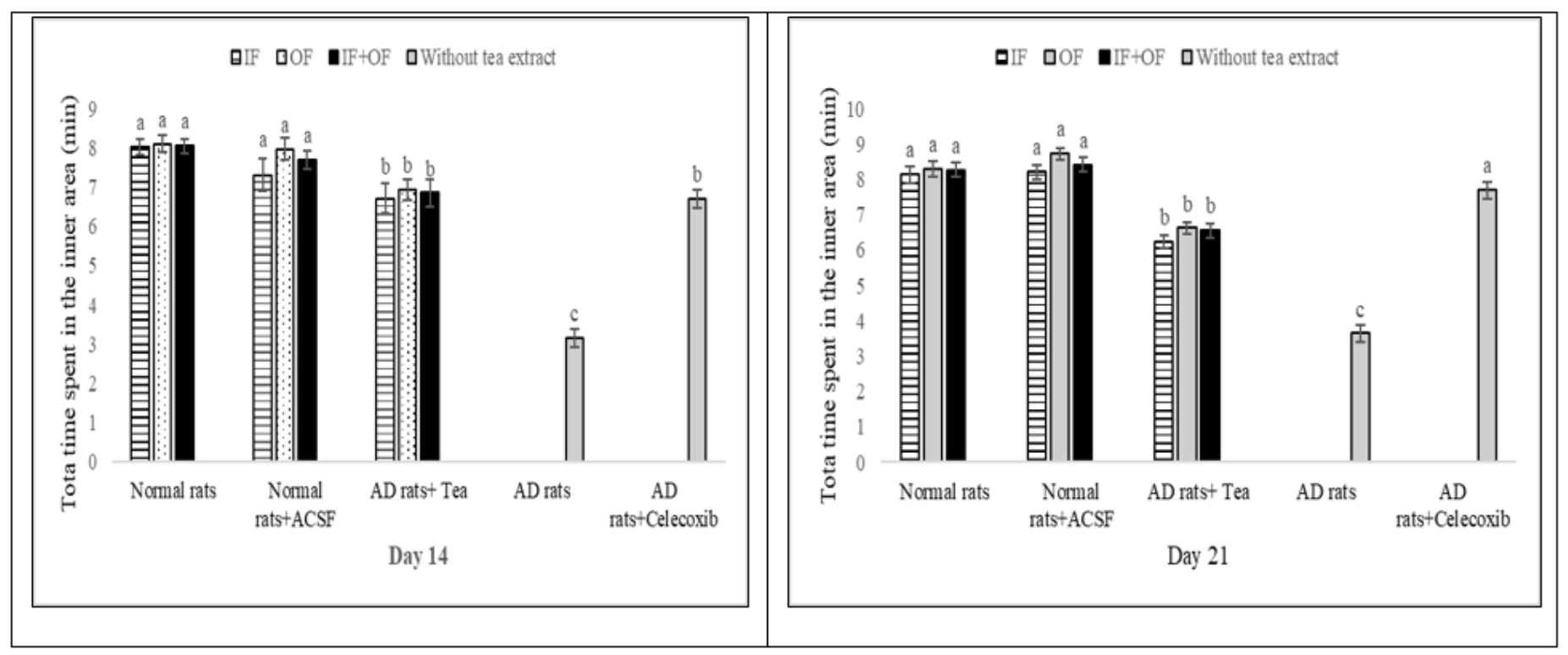

Figure 3

Impact of tea extracts of different field level nutrient management and celecoxib on amount of time spent in the inner area in different experimental groups of rats 


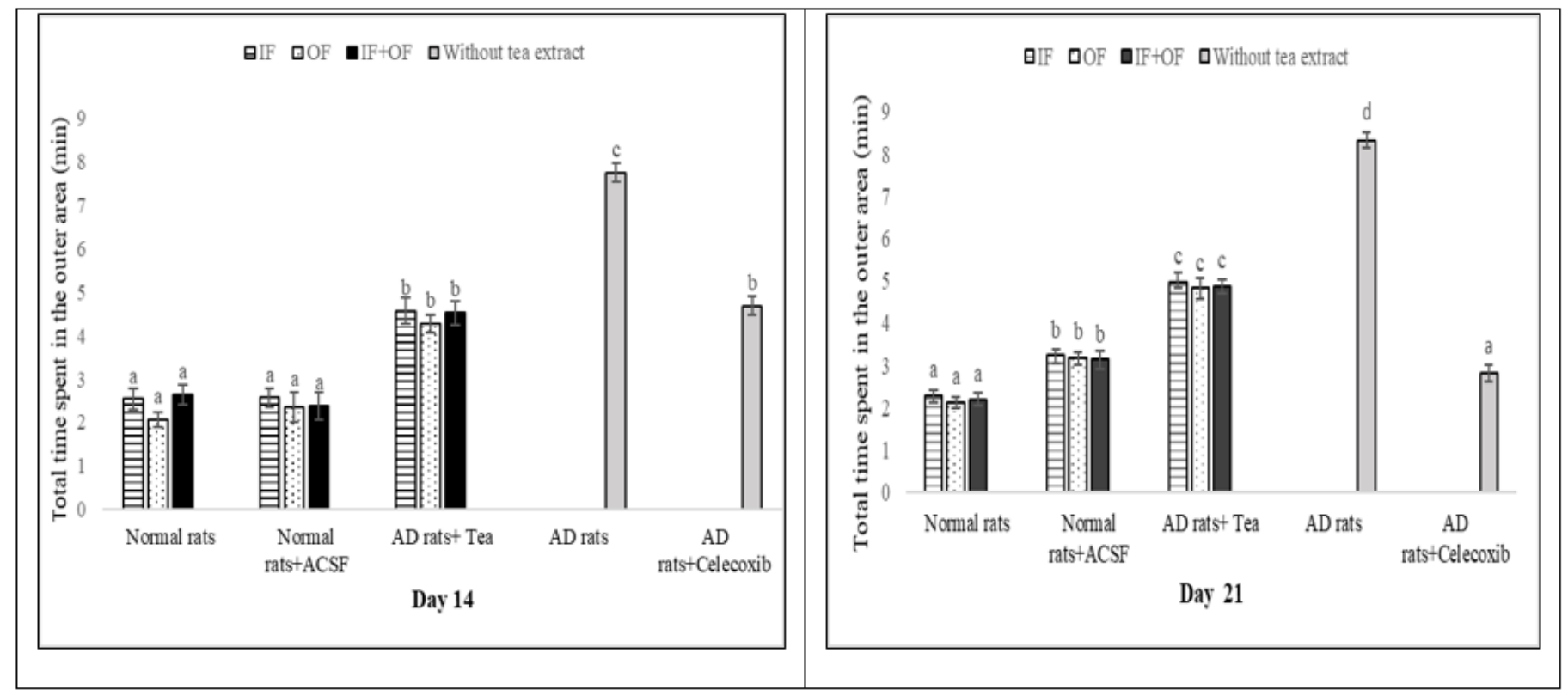

Figure 4

Impact of tea extract of different field level nutrient management and celecoxib on amount of time spent in the outer area in different experimental groups of rats

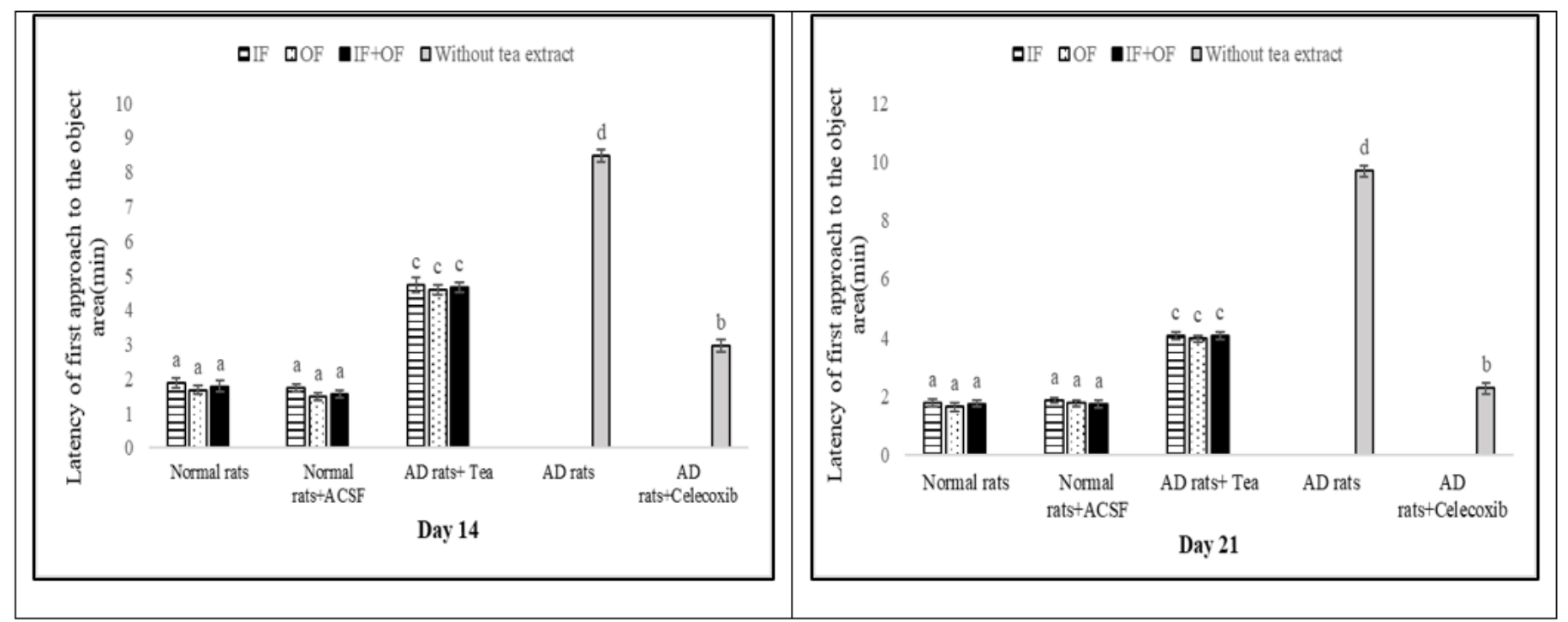

Figure 5

Impact of tea extract of different field level nutrient management and celecoxib on latency of first approach to the object area in different experimental groups of rats 


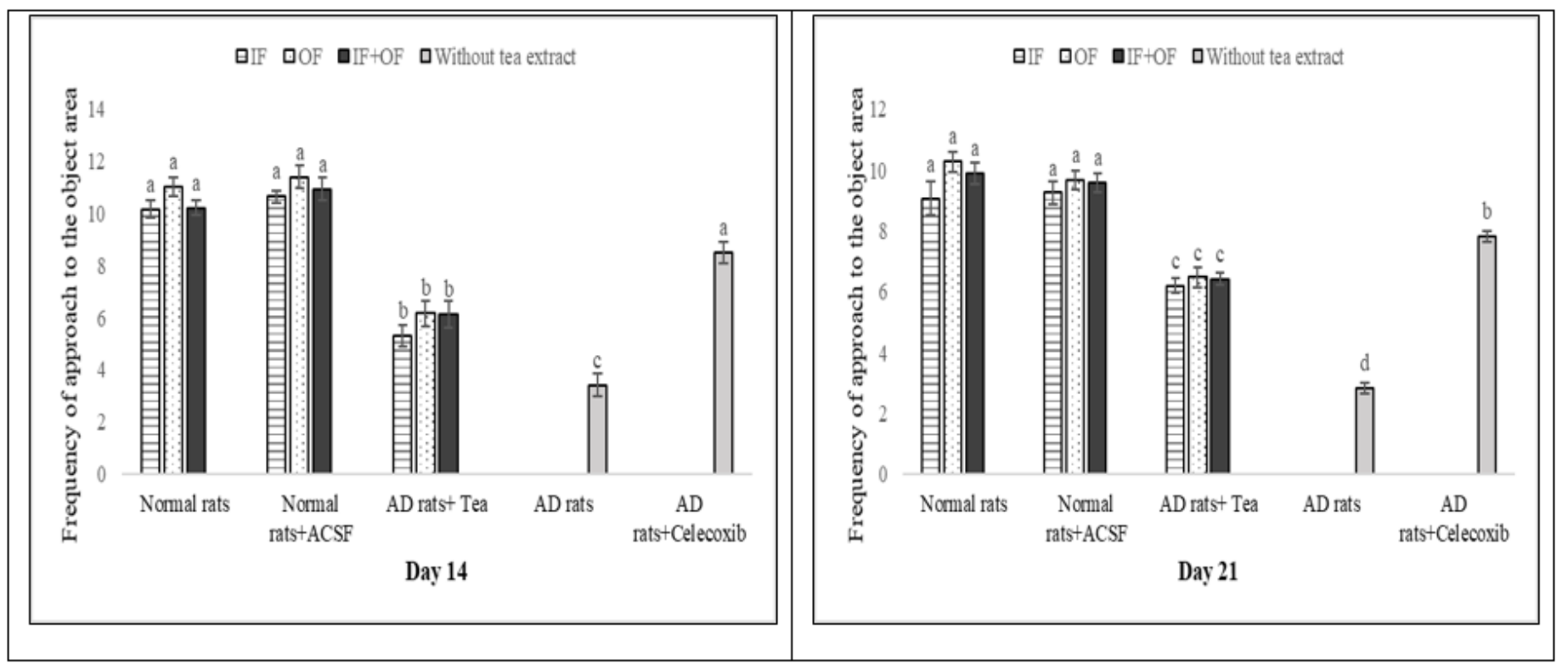

Figure 6

Impact of tea extract of different field level nutrient management and celecoxib on frequency of approach to the object area in different experimental groups of rats

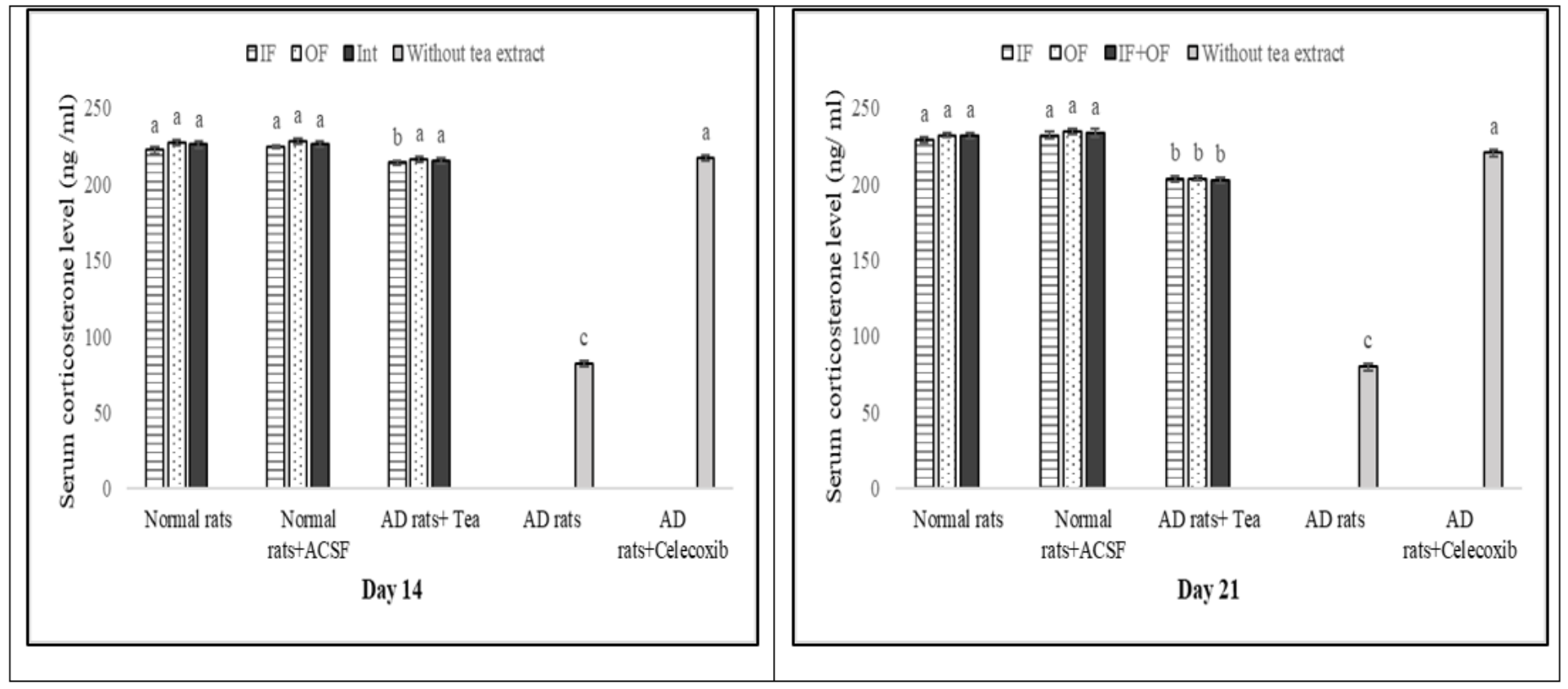

Figure 7

Impact of tea extract of different field level nutrient management and celecoxib on serum corticosterone level in different experimental groups of rats

\section{Supplementary Files}


This is a list of supplementary files associated with this preprint. Click to download.

- Image1.docx

- Image2.docx 